\title{
Globale Probleme einer regionalen Allianz: die NATO und die Frage militärischen Engagements außerhalb der Bündnisgrenzen bis 1989
}

\author{
Bernd Lemke*
}

\begin{abstract}
Already in the early history of NATO, there were differences with respect to the regional scope of the alliance. The old colonial powers, especially France, Great Britain, Portugal and Belgium tried to summon direct or indirect support from NATO to stabilize their empires against the rising indigenous movements. While the US was prepared to give at least some logistic or financial assistance from national resources, NATO as a whole refused to leave the regional framework. Not least Germany and the Scandianavian members regularly critisized all "out-of-area" engagements. Later on, the US was intrested in systematically involving NATO in its worldwide military activities but had little success in convincing the Europeans. The historical analysis indicates a degree of continuity in debates on whether NATO is a regional organisation or one with global reach.
\end{abstract}

Keywords: NATO, Cold War, foreign military missions, transatlantic relationship

NATO, Kalter Krieg, Auslandseinsätze, transatlantisches Verhältnis

\section{Einleitung}

Im Jahre 2008 stellt sich mehr denn je die Frage, ob die NATO den globalen Herausforderungen der Zeit gewachsen ist bzw. ob sie diesen Problemen gewachsen sein will. Das Engagement in Afghanistan zeigt deutlich die immer noch bestehenden Grenzen der nordatlantischen Allianz auf. Nicht nur gibt es fortgesetzte Streitigkeiten um Art und Umfang der genuin militärischen Beiträge im Land selbst, sondern auch Unklarheit über die politische Bewältigung der tiefer liegenden Ursachen.

Damit stellt sich nicht allein die Frage nach der Ausdehnung der geografischen Grenzen für Engagements der NATO, sondern nach dem Grundcharakter der Allianz generell. Es steht zu erwarten, dass es mit einer rein ,quantitativen' Ausdehnung der strategischen Reichweite der NATO nicht getan sein wird, sondern dass sich das Bündnis qualitativ ändern muss, um dauerhaften Erfolg zu haben.

Um die Dimensionen einer solchen Änderung zu beleuchten, hilft ein Blick auf die Geschichte der NATO in Bezug auf die Frage von „out-of-area“-Einsätzen. Zunächst soll die politische Geschichte der „out-of-area“-Problematik und vor allem die Rolle verschiedener Mitglieder darin beleuchtet werden. Danach wird die militärische Seite solcher Einsätze dargestellt. Dadurch sollen die Grenzziehungen der NATO so genau wie möglich identifiziert werden. Behandelt werden dabei insbesondere drei Formationen: die Allied Mobile Force (AMF), die Standing Naval Force Atlantik (SNFL) und die Rapid Deployment Joint Task Force (RDJTF).

\footnotetext{
* Dr. Bernd Lemke, Potsdam, ist Wissenschaftlicher Mitarbeiter des Militärgeschichtlichen Forschungsamtes in Potsdam
}

\section{Die „out-of-area“-Problematik als politischer Sprengstoff für die Bündniskohärenz}

Die Frage nach der geografischen Reichweite der nordatlantischen Allianz stand von Anfang an im Zentrum der Überlegungen. In den Vorgesprächen zur Gründung der NATO 1948/49 wurden verschiedene Modelle für deren Gestaltung vorgestellt und Vorschläge zum Aufbau eines zumindest teilweise global ausgerichteten Paktes gemacht. Sie ließen sich nicht verwirklichen, weil maßgebliche Kräfte, unter ihnen auch die USA, eine Beschränkung der NATO auf den Nordatlantik befürworteten. ${ }^{1}$ Ferner plädierten insbesondere auch die alten Kolonialmächte Großbritannien und Frankreich trotz ihrer globalen Ambitionen keineswegs für eine entsprechende Ausrichtung der NATO. Während der Verhandlungen zur Gründung der UN stimmten sie gegen eine umfassende Ausbreitung des Geltungsbereichs von Bündnissen, da sie fürchteten, dass es zu einer raschen Etablierung eines Globalmanagements durch die USA und die Sowjetunion kommen bzw. ein Scheitern globaler Friedensregelungen zur Destabilisierung des internationalen Systems führen könnte. ${ }^{2}$ So kam es zur Verabschiedung der auch für die NATO bedeutsamen Artikel 51 und 52 in der UN-Charta, die die kollektive Selbstverteidigung im regionalen Rahmen erlaubten. Diese Begrenzung ließ den mittleren Mächten die Möglichkeit einer Einschränkung der Handlungsfreiheit auch des Hegemons.

Diese Engfassung bedeutete nun allerdings keineswegs, dass alle Mitgliedstaaten, auch nicht Großbritannien und Frankreich, diese Grenzen als unabänderlich betrachteten. Frankreich hatte etwa trotz erheblicher Bedenken Washingtons durchsetzen

1 Douglas Stuart und William Tow, The Limits of Alliance, NATO Out-of-Area Problems since 1949, Baltimore London 1990, S. 5f. und 251.

2 Ebda., S. 25 
können, dass Algerien in Artikel 6 des Nordatlantikvertrags explizit als Teil des Bündnisgebiets bezeichnet wurde. ${ }^{3}$

Je nach Interessenlage kam zudem in den nachfolgenden Jahren zu intensiven Bemühungen unterschiedlicher Mitgliedstaaten, das Bündnisgebiet zu erweitern bzw. die Allianz zur Beteiligung oder zumindest zur Unterstützung globaler Einsätze zu bewegen.

Bei ihrer Gründung gar nicht als mögliches Bündnisgebiet in Erwägung gezogen, traten bis 1952 Griechenland und die Türkei der NATO bei - Schritte, die für die weitere Entwicklung der Allianz entscheidend waren und für die strategischen Grundperspektiven bis heute von erheblicher Bedeutung sind. ${ }^{4}$

Die Grundausrichtung der NATO änderte sich bis zum Ende des Ost-West-Konflikts indes kaum. Auch im globalen Zusammenhang bildete die regionale Ausrichtung weiterhin das entscheidende Merkmal von Militärbündnissen.Dies galt für die Central Treaty Organisation (CENTO = Bagdad-Pakt) und den ANZUS-Pakt, der schließlich in die South East Asia Treaty Organisation (SEATO) einmündete. ${ }^{5}$ Diese Bündnisse bildeten aus Sicht der USA gewissermaßen „handhabbare“ Teilelemente ohne formalen Gesamtrahmen. Bezeichnenderweise zerfielen sie infolge der Schwäche und des fehlenden Zusammenhalts ihrer Mitglieder recht rasch - eine Tatsache, die für die Bewertung des Nutzens eines stärkeren Engagements der NATO außerhalb ihrer Bündnisgrenzen von nicht zu unterschätzender Bedeutung ist.

Auch die Geschichte der praktischen Problemfälle ist so alt wie die NATO selbst. Bereits zwischen 1950 und 1953 entstand ein Konflikt um die Gestaltung der Machtverhältnisse im Mittleren Osten, dies nicht zuletzt auch vor dem Hintergrund der Probleme an der NATO-Südflanke. Die britische Regierung, darauf bedacht, den eigenen Einfluss trotz schwindendem Machtpotenzial Großbritanniens zu wahren, schlug den Aufbau eines Middle East Command (MEC) bzw. einer Middle East Defence Organisation (MEDO) vor, die ggf. auch Griechenland und die Türkei einbeziehen sollte. ${ }^{6}$ Gemäß ihrer strategischen Grundlinie versuchte sie, eine „special relationship“ mit den USA zu etablieren, gewissermaßen mit ihnen zusammen eine Hegemonie zu errichten. Die Amerikaner waren zwar grundsätzlich an dieser Zusammenarbeit interessiert, Großbritannien entwickelte sich aufgrund seiner unverändert imperial ausgerichteten und repressiven Politik in der Region (z.B. gegenüber Nasser) jedoch mehr und mehr zu einer Belastung, ohne Substanziel-

3 Christopher Coker, NATO and Africa 1949 - 89: An Overview, in: Gustav Schmidt (Hrsg.), A History of NATO - The First Fifty Years, Vol. 1, Houndmills 2001, S. 153. Der entsprechende Passus wurde interessanterweise zwar außer Kraft gesetzt, aber bis heute formal nicht aus dem Vertrag getilgt.

4 Zum Beitritt von Türkei und Griechenland zur NATO vgl. insbesondere S. Victor Papascoma, Greece and NATO, A Nettlesome Relationship, in: Gustav Schmidt (Hrsg.), A History of NATO - The First Fifty Years, Vol. 3, Houndmills 2001, S. 359f. und Bruce Kuniholm, The Evolving Strategic Significance of Turkey's Relationship with NATO, in: ebda., S. 339 - 347.

5 Zu den genannten Bündnisse vgl. Stuart/Tow, The Limits of Alliance, S. 51 - 53, 114 - 150. Vgl zusätzlich Behcet Kemal Yesilbursa, The Baghdad Pact, Anglo-American Defence Policies in the Middle East 1950 - 1959, London 2005.

6 Stuart/Tow, The Limits of Alliance, S. 51f. Vgl. auch Douglas T. Stuart, The United States and NATO Out-of-Area Disputes, in: Gustav Schmidt (Hrsg.), A History of NATO - The First Fifty Years, Vol. 1, Houndmills 2001, S. 126. Die NATO hatte das Projekt nur halbherzig unterstützt. Frode Liland, Explaining NATO's Non-Policy on Out-Of-Area Issues during the Cold War, in: Gustav Schmidt (Hrsg.), A History of NATO - The First Fifty Years, Vol. 1, Houndmills 2001, S. 179. les beitragen zu können. So war dieses Projekt zum Scheitern verurteilt.

Nach ähnlichem Muster verliefen auch alle weiteren Konflikte und Krisen, zu nennen sind der französische Indochinakrieg, der Aufstand in Algerien und die Suezkrise. ${ }^{7}$ Die alten Kolonialmächte, inzwischen viel zu schwach, um ihre Besitzungen ohne fremde Hilfe erhalten zu können, traten an die USA und die NATO heran, um militärische, politische oder zumindest ökonomische Unterstützung zu erhalten. Dabei führte insbesondere Frankreich die heute wieder recht modern klingende Begründung an, durch ihre Bemühungen um koloniale Stabilisierung würde die Sicherheit vor allem auch der NATO gewährleistet. Die Allianz könne sich nicht einfach auf ihre formalen Grenzen zurückziehen, wenn ein globaler „guerre revolutionaire" tobe, der zudem noch von der Sowjetunion aktiv befeuert werde.

Frankreich konnte zwar die amerikanische Regierung dazu bewegen, finanzielle und logistische Unterstützung zu leisten, die meisten anderen NATO-Mitglieder, hier vor allem die Bundesrepublik und die skandinavischen Staaten, blieben von der französischen Argumentation jedoch unbeeindruckt. ${ }^{8}$ Neben strategischen Erwägungen, vor allem der unmittelbaren Bedrohung durch die Armeen des Warschauer Paktes, spielten hierbei auch politisch-moralische und vor allem politisch-ökonomische Überlegungen eine wichtige Rolle. Demnach war es keineswegs ausgemacht, dass die Kolonialmächte, neben Frankreich und Großbritannien vor allem Belgien und Portugal ${ }^{9}$, durch ihr hartnäckiges Festhalten an ihren imperialen Besitzungen zur Stabilität der NATO beitrugen. Durch die Unterdrückung der entstehenden Nationalbewegungen würden die betroffenen Regionen eher destabilisiert und das Ansehen des Westens geschädigt. Hinzu kam der Einsatz erheblicher militärischer und ökonomischer Mittel in den Kolonialgebieten zu Lasten der Verteidigungsaufwendungen für Europa. Die militärische Führung der Bundesrepublik betrachtete nicht zuletzt aufgrund der exponierten Lage des Landes in Europa-Mitte alle noch so begrenzten Versuche, Truppen aus dem Befehlsbereich des SACEUR abzuziehen, mit Misstrauen und Kritik.

Diese Interessendivergenzen führten schließlich zu einer Zerreißprobe für die NATO. Frankreich, das 1954 nicht zuletzt wegen vorgeblich fehlender Unterstützung durch die Allianzpartner in Indochina bereits den EVG-Vertrag hatte scheitern lassen, gelangte zu der Überzeugung, dass das Bündnis für das eigene Land kaum Nutzen brachte und zog sich schließlich 1966 aus den Militärstrukturen der Allianz zurück. Die NATO geriet in ihre bis dahin schwerste Existenzkrise.

In den 1960er Jahren kam es nicht zuletzt infolge der Niederlagen der alten Kolonialmächte zu einem grundsätzlichen Rollentausch. Die USA übernahmen nun in gewisser Weise die Politik Frankreichs und Großbritanniens. ${ }^{10}$

\footnotetext{
7 Zu Frankreich vgl. Stuart/Tow, The Limits of Alliance, Teil III.

8 Ebda., Teil IV.

9 Christopher Coker, NATO, the Warsaw Pact and Africa, Houndsmill 1985. Kap. $3-5$ und 10.

10 Frode Liland, Explaining NATO's Non-Policy on Out-Of-Area Issues during the Cold War, S. $181-185$.
} 
Die bündnisinternen Konflikte wiederholten sich mutatis mutandis, nur dass nunmehr die USA versuchten, die NATO zu größerem Engagement für ihre Politik außerhalb des Bündnisgebiets zu bewegen. Dies galt insbesondere für den Krieg in Vietnam und den Mittleren Osten, der ab 1973 immer stärker in den Fokus geriet und infolge der Ereignisse in Afghanistan und im Iran 1979 die amerikanische Sicherheitspolitik unter der Präsidentschaft Jimmy Carters weitgehend bestimmte. ${ }^{11}$ Die Reagan-Administration initiierte schließlich eine Neuorientierung der amerikanischen Miliärstrategie mit dem Ziel einer deutlichen Erhöhung der Flexibilität der Streitkräfte für Einsätze auf dem gesamten Globus.

Insgesamt aber änderte sich die Haltung in der NATO hinsichtlich „Out-of-area“-Einsätzen nicht. Sie blieben weiterhin Sache einzelner Nationalstaaten, nunmehr vor allem der USA. Zum fortgesetzten Ärger und Verdruss der Vereinigten Staaten und insbesondere deren Außenminister Henry Kissinger, verweigerten die NATO-Partner ihnen jegliche substanzielle Unterstützung für Einsätze außerhalb des Bündnisgebiets. Frankreich und Großbritannien taten dies nicht zuletzt auch aus Bitterkeit wegen der ihnen zuvor von Washington verweigerten Hilfen. Allerdings fehlten ihnen dazu auch die erforderlichen Mittel.

Deutliche Meinungsverschiedenheiten und Konflikte innerhalb der NATO kennzeichneten auch die 1980er Jahre. Die USA, ähnlich wie die Kolonialmächte zuvor enttäuscht von den Allianzpartnern, griffen schließlich zur Selbsthilfe und schufen vollendete Tatsachen - letztlich auch ohne Zustimmung der Europäer.

\section{An der Peripherie und darüber hinaus: mobile Einsatzverbände als Manifestation der geogra- fischen Grenzen der Allianz}

Um näher zu analysieren, wie genau es die NATO mit der Einhaltung der selbstgesetzten Schranken nahm und die Tragweite der damit verbundenen Konflikte zu ergründen, hilft ein Blick auf die Geschichte wichtiger mobiler Einsatzverbände des Bündnisses bzw. der USA. ${ }^{12}$ Untersucht werden in der Folge die Allied Mobile Force, AMF (L) und (A), eine spezielle Einsatztruppe der NATO zur Abschreckung von begrenzten feindlichen Aktionen an neuralgischen Punkten an den Flanken, die Standing Naval Force Atlantic (SNFL oder auch STANAVFORLANT), gewissermaßen das Marineäquivalent zur AMF, das als mobiler Flottenverband vor allem in der Nordsee, im Nordmeer und im Atlantik den Willen der NATO demonstrieren sollte, keinerlei Provokationen oder Aggressionen des Warschauer Pakts zur See kommentarlos hinzunehmen. Die Rapid Deployment Joint Task Force (RDJTF), ein rein amerikanischer Verband zum Einsatz im Arabischen Golf, soll im Folgenden als Kontrast zu den beiden anderen Verbänden vorgestellt werden, um zu demonstrieren, wo die Grenzen der NATO in Bezug auf Ressourcen und geografischem Einsatzgebiet genau lagen.

11 Stuart/Tow, The Limits of Alliance, S. 88 - 99.

12 Die im Folgenden dargestellten Einsatzverbände umfassten nicht alle zur Verfügung stehenden Truppen. Nationale Verbände der europäischen Hauptmächte, beispielsweise die britische Verstärkung für Europa-Mitte oder andere Gebiete, die UK Mobile Force (UKMF) oder die französische Force d'Action Rapide (FAR), können aus Platzgründen nicht mitbehandelt werden.
Die AMF wurde Ende der 1950er Jahre konzipiert und aufgebaut. Ihre Anfänge reichen nach bisherigem Forschungsstand zurück bis zu den ersten Initiativen zum Wechsel von der Strategie der „massive retaliation“ zur „flexible response“. Hintergrund war die Erkenntnis, dass das Bündnis auf lokale Provokationen bzw. Versuche des Warschauer Pakts zur Desintegration mit einer nuklearen Vergeltungsdrohung nicht glaubwürdig reagieren konnte. ${ }^{13}$ Die Verbände der AMF (ingesamt sechs Bataillone [AMF/L] mit je einer Jabo-Staffel [AMF/A]) bestanden aus hochmobilen Eliteeinheiten, die in der Lage waren, Spezialaufgaben in Grenzregionen und -situationen durchzuführen. Ihre Einsatzgebiete als ,Feuerwehr' der NATO lagen ausnahmslos an neuralgischen Punkten an den äußersten Linien der Allianz. Insbesondere an den Flanken des Bündnisses bestand die Gefahr, durch „incursions“, „infiltrations“ oder „local hostile actions“ die Kontrolle zu verlieren. Solche Aktionen reichten von Provokation im Vorfeld eines möglichen Großkrieges bis hin zur langfristigen, systematischen Aufweichung der Peripherie. Besonders gefährdet erschienen hierbei Nordnorwegen, Istrien, die Nordgrenze der Türkei bzw. Griechenlands zu Bulgarien sowie der Osten und der Süden der Türkei.

Die AMF, an der sich alle NATO-Partner beteiligten, sollte dazu dienen, diese Gefahren von Beginn an einzudämmen und zusätzlich Bündnissolidarität zu demonstrieren. ${ }^{14}$ Im Konfliktfall hätte jeder Mitgliedstaat in der Pflicht gestanden. Gemäß Artikel 5 des Nordatlantikvertrags hätte die NATO selbst nicht den Verteidigungsfall ausrufen können. Dies war vielmehr Sache der einzelnen Mitgliedstaaten. ${ }^{15}$

Der Gedanke liegt nahe, dass die AMF zumindest kurzfristig und punktuell ggf. auch jenseits des NATO-Bündnisgebiets hätte eingesetzt werden können. Die Furcht vor einer Eskalation mit einem nachfolgendem nuklearen Schlagabtausch war jedoch derart groß, dass die NATO-Planer alles vermieden, was zu einem solchen Kontrollverlust führen konnte. Die Einsatzanweisungen („Rules of Engagement“) der AMF enthielten aus diesem Grund ein ausführliches, äußerst restriktiv angelegtes

$13 \mathrm{Zu}$ den Hintergründen der strategischen Entwicklung vgl. grundsätzlich Bruno Thoß, NATO-Strategie und nationale Verteidigungsplanung, Planung und Aufbau der Bundeswehr unter den Bedingungen ener massiven atomaren Vergeltungsstrategie 1952 bis 1960, München 2006, S. 513 - 601.

$14 \mathrm{Zu}$ den Anfängen der AMF ist in den elektronischen Publikationen des IMS (NATO), hier insbesondere im Public Disclosure Programm (PDP) aussagekräftiges Material vorhanden. In diesem Zusammenhang nur die beiden wichtigsten Dokumente: IMS, PDP, CD 010, Allied Command Europe Mobile Forces, 13.6.61, MCM-080-61_ENG_PDP.pdf, IMS, PDP, CD 011, ACE Mobile Force 19.1.1965, MCM-014-65_ENG_PDP.pdf, IMS. Vgl. zusätzlich die innerhalb des PDP herausgegebene CD mit den Akten der Working Group AC/212 (Financing of the ACE Mobile Force Exercises 1961 67). Zur Gesamtgeschichte gibt es bislang kaum Publikationen. Vgl. Maloney, Sean M., Fire Brigade or Tocsin? NATO's ACE Mobile Force, Flexible Response and the Cold War, in: The Journal of Strategic Studies, Vol. 27, No. 4, Dez. 2004, S. 585 - 613 und Steinle, Götz, Allied Mobile Force, Ein Mittel zur Krisenbewältigung, in: Truppenpraxis 2/1991, S. 121 - 125. Im Militärgeschichtlichen Forschungsamt entsteht derzeit eine Studie zu AMF und STANAVFORLANT.

15 Artikel 5 NATO-Vertrag legt für jede Mitgliedsnation lediglich fest, das sie "<...> der Partei oder den Parteien, die angegriffen werden, Beistand leistet, indem jede von ihnen unverzüglich für sich und im Zusammenwirken mit den anderen Parteien die Maßnahmen, einschließlich der Anwendung von Waffengewalt, trifft, die sie für erforderlich erachtet, um die Sicherheit des nordatlantischen Gebiets wiederherzustellen und zu erhalten."

Vgl. dazu auch das Urteil des Bundesverfassungsgerichts vom 3. Juli 2007 zum ISAF-Einsatz. Danach begründet Artikel 5 NATO-Vertrag „völkerrechtlich eine Beistandspflicht $\langle\ldots\rangle$, die die Mitgliedstaaten in eigener Verantwortung ausfüllen“. BVerfG, 2 BvE 2/07 vom 3.7.2007, Absatz-Nr. 52, http://www.bverfg. de/entscheidungen/es20070703_2bve000207.html. 
Regelwerk, das jeden einzelnen Schritt genau definierte. ${ }^{16}$ Die Stäbe waren sich dabei einig, dass die AMF nur in begrenztem Maße, eben gewissermaßen als ,Speerspitze' und als Element einer frühzeitigen Abwehr militärische Wirkung erzielen konnte. Geplant war, sie im Falle eines bevorstehenden Großkrieges („general war“) unverzüglich von den Flanken zurückzuziehen. Letztlich bestand ihre Aufgabe aber vor allem drin, eine derartige Entwicklung durch Präsenz im Frieden von vornherein zu vermeiden. Daher wurden auch jedes Jahr öffentlichkeitswirksame Übungen veranstaltet, die von einem massiven Presse- und Propagandaaufwand begleitet wurden. Zu operativen Einsätzen der AMF ist es während des Ost-West-Konflikts nicht gekommen, obwohl die Planer solche Einsätze in einigen kritischen Situationen durchaus ins Auge gefasst hatten. ${ }^{17}$ Das erste wirklich Ereignis von Belang war die Verlegung der Luftkomponente der AMF (A) in die Türkei während des Golfkrieges 1990/91.

Insgesamt war die AMF fester, integraler Bestandteil des Abschreckungs- und Eskalationsinstrumentariums der NATO. ${ }^{18}$ Nicht zuletzt auch aufgrund der bereits beschriebenen prinzipiell defensiven Haltung vieler Mitglieder wäre auch die ernsthafte Planung eines Einsatzes außerhalb des Bündnisgebiets vollkommen unmöglich gewesen.

Die STANAVFORLANT war zunnächst als Schutz- und Versorgungsverband der AMF vor allem in Norwegen gedacht. ${ }^{19}$ Recht rasch erhielt sie jedoch andere, weiterreichende Aufgaben. ${ }^{20}$ Ausgehend von der Vorstellung, dass allen lokalen Provokationen und feindseligen Akten des Warschauer Paktes von Beginn an entgegengetreten werden musste, wurde ein multilateraler Fregatten- und Zerstörerverband zusammengestellt, der sich durch hohe Mobilität auszeichnete. $\mathrm{Zu}$ den Bedrohungsszenarien zählten Provokationen oder Übergriffe gegen die zivile Schifffahrt von Bündnismitgliedern, also etwa gegen Fischereioder Handelsschiffe, insbesondere in der nördlichen Nordsee, oder gegen die empfindlichen Nachschublinien im Nordatlantik. In Betracht gezogen wurden auch provokative Aktionen des Paktes wie etwa eine verstärkte Präsenz von Kriegsschiffen vor den Küsten der NATO-Mitgliedstaaten (etwa in der Nordsee) oder an neuralgischen Punkten. ${ }^{21}$ Wie bei AMF-Operationen herrschte auch hier die Furcht vor einer schnellen Eskalation, und so galt für SNFL-Einsätze in Krisensituationen ebenfalls ein restriktiver und genau ausformulierter Katalog an Verhaltensmaßregeln („Rules of Engagement“). ${ }^{22}$

16 SHAPE-Archiv Mons, 16 mm P01-B R-54 L-597, HQ Defence Command North Norway Oplan 21421, Reception and Employment of AMF Land Component (Bland Beer), 10.5.78, 2000007443.pdf, App. 1 to Ann.E.

17 So etwa im Falle eines Einmarsches der Armeen des Warschauer Paktes in Polen 1980. BA-MA, BW 2 / 13419, Verschlusssache.

18 Die entscheidenden Wegmarken der strategischen Entwicklung sind zu finden: für die MC 14 und die MC 48 in ihren Versionen bis 1968 in: NATO Strategy Documents $1949-1969$, hrsg. von Gregory W. Pedlow in collaboration with NATO International Staff Central Archives, o.O., 1997. Komplett downloadbar unter http://www.nato.int/archives/strategy.htm. Die MC 70 in: IMS, PDP, CD 003, MC 70 ENG PDP.pdf.

19 IMS, PDP, CD 011, MCM-133-65_ENG_PDP.pdf, Naval Element for ACE Mobile Force, vom 17.9.1965.

20 Zum Folgenden vgl. grundsätzlich und soweit nicht anders angegeben BAMA, BW 2 / 9057, 11119 und 13419, mehrere Verschlusssachen.

21 BA-MA, BW 2 / 9057, Fü S III 6, Vortragsnotiz für StAL Fü S III, Möglicher Einsatz der STANAVFORLANT zur Überwachung in der Nordsee übender sowjetischer Streitkräfte, 29.3.77. Begleitdokumente.

22 Ebda.
Hauptaufgabe war auch hier militärische Präsenz und die Demonstration von Bündnissolidarität im Frieden. Entsprechend häufig befand sich die SNFL auf See - in weitaus höherem Maße als bei den Hauptkampfverbänden der NATO üblich - und bereiste alle wichtigen westlichen Seehäfen. Dazu gehörten auch Besuche im Mittelmeer und in Portugal. ${ }^{23}$ Der Verband steuerte nach einem festgelegten Plan innerhalb eines Jahres alle Bündnisstaaten an. Auf See und in den Häfen erhielt die SNFL häufig Besuche und Begleitung durch zusätzliche Marineeinheiten oder Vertreter von Regierungen und Verwaltungen, sie nahm an Marineübungen teil und führte Austauschprogramme durch. Ferner wurde auch hier auf umfangreiche Öffentlichkeitsarbeit gesetzt, um zu demonstrieren, dass die NATO durch die SNFL in der Lage war, auf feindliche Vorstöße oder Provokationen rasch zu reagieren.

Angesichts der "Unteilbarkeit" des Atlantiks und der Weltmeere stellte sich auch für die SNFL die Frage nach einer Ausdehnung des Einsatzgebiets. Dies galt nicht zuletzt auch vor dem Hintergrund massiver Forderungen der obersten Kommandobehörden nach einer zusammenhängenden Verteidigung zum Schutz der Seeverbindungen. Insbesondere in den 1980er Jahren nahm das Bedrohungsgefühl hier erheblich zu, die Krisen im Nahen Osten und die stärker werdende Präsenz der sowjetischen Marine rund um Afrika deuteten auf die Verwundbarkeit der Ölversorgung des Westens. SACLANT erstellte auf Anweisung des Militärausschusses deshalb Contingency Plans für den Schutz des Südatlantiks, ferner wurden grundlegende Operation Plans für den Schutz der NATO-Handelsmarinen erstellt („Plan Limmer“). ${ }^{24}$ Darin waren ausführliche Anweisungen für den Schutz von Handelsschiffen (z.B. Überwachung oder Zusammenstellung von Konvois in ausgewählten Häfen) enthalten, dies allerdings unter strikter Wahrung der nationalen Prärogativen und unter Achtung der Entscheidungsfreiheit von Privateigentümern - zumindest solange noch keine Feindseligkeiten ausgebrochen waren.

Auch hier griffen jedoch die Selbstbeschränkungen der Allianz. Im NATO-Vertrag war der Wendekreis des Krebses als Südgrenze des Operations- und Einsatzgebiets angegeben worden. Diese Linie wurde nicht überschritten, obwohl auch Häfen außerhalb des NATO-Gebiets als Sammel- und Kontrollpunkte vorgesehen waren (Küsten von Südamerika und Afrika). ${ }^{25}$ Abgesehen davon war für NATO-Kriegsschiffe selbst in den weitestgehenden Planungen nie die Erlaubnis enthalten, in die Küstengewässer von Nicht-NATO-Staaten einzulaufen.

Ein besonders heikler Punkt in diesem Zusammenhang war die Rolle Südafrikas, die für die Frage nach den NATO-Grenzen zeitweise bedeutsames Gewicht besaß. Sie kann an dieser Stelle

23 Vgl. z.B. BA-MA, BW 2 / 11119, Fü S III 6 an dmv/mc, Operationen der STANAVFORLANT in der Ostsee und im Mittelmeer, 2.3.79. Ausführliche Begleitdokumente.

24 BA-MA, BW 2 / 9057, Fü M II 1, SACLANT Operation Plan Nr. 114-70 (Protection of NATO Fishing Vessels), 17.8.70, Ebda., Fü S III 6 an dmv / MC, Naval Control of Merchant Shipping - Plan Limmer, 13.11.76. Teils ausführliche Begleitdokumente.

25 Diese Aussage besitzt aufgrund der noch im Gang befindlichen Quellenforschungen und der Geheimhaltungsbestimmungen einstweilen noch bedingte Gültigkeit. Insbesondere ist noch zu untersuchen, inwieweit sich die NATOStaaten tatsächlich mit Planungen und Allokation von Ressourcen südlich des Wendekreises des Krebses beschäftigten. Die durchgesehenen Akten deuten an, dass hier kaum wirkliche Initiativen mit praktischen Folgen ergriffen worden sind. 
jedoch aus Platzgründen nur angerissen werden. ${ }^{26}$ Südafrika, das fortgesetzte Versuche unternahm, Mitglied der NATO zu werden oder zumindest als externer Partner anerkannt zu werden, erhielt hierbei zumindest zeitweise die Unterstützung konservativer Militärplaner und -publizisten in Großbritannien. Großbritannien schloss schließlich zeitweise Nutzungsverträge mit Südafrika ab („Simonstown-Agreement“), zudem unterhielten die westlichen Staaten offenbar auch eine elektronische Überwachungsanlage in Silvermine, nahe bei Simonstown. ${ }^{27}$ Hinzu kamen Waffen- und Technologielieferungen. Aufgrund der Apartheidpolitik und der militärischen Operationen Südafrikas in den Nachbarstaaten wurde diese Art der Zusammenarbeit jedoch bald eingestellt. Derartige Aktivitäten jenseits des Bündnisgebiets gingen ohnehin über den Umfang anderer, von vielen Mitgliedstaaten bereits abgelehnter Projekte hinaus. ${ }^{28}$

Die Frage nach einem eigenständigen Schutz der Handelsflotten der Mitgliedstaaten durch die NATO stellte sich auch in der Folgezeit noch. Insbesondere die SNFL geriet aufgrund ihrer Mobilität in den Blick, allerdings verfügte sie mit ca. sechs mittleren Kriegsschiffen kaum über die nötige Stärke für die Übernahme großangelegter Schutzfunktionen im Atlantik oder gar im Indischen Ozean. Die deutschen Stellen etwa sahen sich lediglich in der Lage, eine Fregatte für Überwachungs- und Verteidigungsaufgaben im NATO-Bereich selbst, also etwa für den Schutz der eigenen Fischereiflotten, abzustellen. Diese war jedoch ohnehin bereits Teil der STANAVFORLANT. ${ }^{29}$ Verwiesen wurde vor allem auf die besondere Lage der Bundesrepublik und der NATO. So wurde in der zweiten Hälfte der 1970er Jahre empfohlen, einen entsprechenden Vorschlag über die Verwendung der Fregatten 122 angesichts der brisanten Thematik und der bevorstehenden Verhandlungen im Bundestag „ruhen zu lassen“. Einige militärische Planer im Führungsstab der Streitkräfte äußerten Frustration angesichts der vorgegebenen Beschränkungen: „Unsere Militärpolitik: mit dem Hut in der Hand unauffällig heranschleichen auf leisen Socken“. ${ }^{30}$

Die einzige „Überschreitung“ des Bündnisgebiets durch die SNFL fand im Rahmen von Manövern vor der Küste der USA statt. Dort sollte sich die SNFL an Seemanövern beteiligen, die auch Puerto Rico einschlossen, das formal außerhalb des Bündnisgebiets lag. Die SNFL sollte während dieser Manöver zu Übungs- und Besuchszwecken das Bündnisgebiet für kurze Zeit verlassen. Obwohl Puerto Rico völkerrechtlich zu den USA

26 Vgl. ausführlich Coker (1985), insbes. Kap. 6 und den auf dieser Studie basierenden Aufsatz von Coker, NATO and Africa 1949 - 89: An Overview, in: Gustav Schmidt (Hrsg.), A History of NATO - The First Fifty Years, Vol. 1, Houndmills 2001, S.153 - 172

27 Coker (1985) , S. 134ff. (Projekt "Advokaat")

28 Die Amerikaner versuchten bis Mitte der 1980er Jahre v.a. durch Verhandlungen zumindest eine regionale Stabilisierung im südlichen Afrika zu erreichen, scheiterten jedoch. Die europäischen Verbündeten kritisierten dabe insbesondere die teilweise Duldung der aggressiven Militäraktionen Pretorias durch die USA. Christopher Coker, The United States and South Africa, 1968 - 1985, Durham 1986, Kap. 11, v.a. S. 228f.

29 BA-MA, BW 2 / 9057, Chef Stab Fü S an Generalinspekteur, Maritime Contingency Forces Atlantik (MARCONFORLANT), 16.11.70, mit weiteren Begleitdokumenten.

30 Hands. Bem. in: BA-MA BW 2 / 9057, Fü S III 1, Contingency-Planung, hier: Schutz der NATO-Handelsschiffahrt südlich des Wendekreises des Krebses, 13.2.76. Ein anderer Planer bezeichnete die Haltung so: „Wasch mir den Pelz, aber mach' mich nicht nass. “ BA-MA, BW 2 / 9057, Fü S III 6, Aktennotiz, Planung zum Einsatz von NATO-Seestreitkräften südlich des Wendekreises des Krebses, 20.2.76. Tiefergehende Informationen, insbesondere für die 1980er Jahre, müssen erst noch durch weitere Forschungen zu Tage gefördert werden. gehörte, rief dies bei manchen NATO-Mitgliedern erhebliches Unbehagen hervor und führte bei den Planungsstellen der Bundeswehr zu besonderen Absicherungsmaßnahmen. Die Operationsabteilungen im Führungsstab der Streitkräfte (Fü S) wurden angwiesen, das Auswärtige Amt zu konsultieren und gaben erst nach einigen Überlegungen und diplomatischen Aktionen grünes Licht. ${ }^{31}$ Der Vorgang macht deutlich, dass hier keinesfalls von einem Versuch zur heimlichen Ausdehnung des NATO-Gebiets gesprochen werden kann.

Insgesamt bestand bei der Bundesmarine aufrund ihrer grundsätzlichen strategischen Ausrichtung nur geringes Interesse, sich auf ein größeres globales Engagement einzulassen. ${ }^{32}$ Das Hauptinteresse richtete sich von Anfang an auf die Sicherung der Ostseezugänge („Baltic Approaches“) und änderte sich zumindest bis zum Ende des Ost-West-Konflikts nicht. Von Seiten der Bundesmarine wurden sogar Anstrengungen unternommen, die SNFL, die eigentlich für ein anderes Einsatzgebiet vorgesehen war, an Übungen in der Ostsee zu beteiligen. Dies gelang allerdings nur in Ausnahmefällen, da einige Bündnispartner, etwa die Niederländer aber auch SACLANT befürchteten, die SNFL könnte in der Folge dauerhaft in die Ostsee entsandt werden. ${ }^{33}$

Die Geschichte der Rapid Deployment Joint Task Force (RDJTF) bildet einen deutlichen Kontrast zur eher von Restriktivität gekennzeichneten Genese von AMF und SNFL, sie deutet dabei zugleich auf Kontinuitäten über das Ende des Ost-WestKonflikts hinaus. ${ }^{34}$ Die Gründe zum Aufbau dieses mobilen Einsatzverbandes finden sich in der zweiten Hälfte der 1970er Jahre, insbesondere nach dem Einmarsch der Sowjetunion in Afghanistan und der Geiselnahme amerikanischer Botschaftsangehöriger in Teheran. Der Nahe Osten galt schon seit dem Yom-Kippur-Krieg von 1973 und dem „Ölembargo“ durch die OPEC als Krisenregion. US-Präsident Carter gab im Jahr 1977 eine Studie für eine Eingreiftruppe in Auftrag, ordnete 1979 deren Aufbau an (zunächst Rapid Deployment Force, RDF) und legte in seinem letzten Regierungsjahr ein strategisches Gesamtkonzept vor, das den Einsatz auch der RDF beinhaltete („Carter-Doktrin“). All dies wurde rasch den skeptischen NATO-Verbündeten präsentiert. Die Planungen und Diskussionen waren bereits weit fortgeschritten, als Ronald Reagan das Amt des amerikanischen Präsidenten übernahm. Während seiner Regierungszeit wurde das Konzept für den Einsatz der Eingreiftruppe (nun „Rapid Deployment Joint Task Force) $)^{35}$ ausgebaut und in gewisser Weise radikalisiert.

31 BA-MA, BW 2 / 9057, Telegramm Fü S III an dmv MC/NATO, Beteiligung STANAVFORLANT an nationaler US-Übung außerhalb des NATO-area (17 - 24 8.74), 13.8.77, BA-MA, BW 2 / 11119, Fü S III 6 an dmv/mc, STANAVFORLANT, out-of-area-operations (5. - 12.8.81), 22.7.81. Jeweils weiteres Begleitmaterial.

32 Zum Folgenden vgl. Johannes Berthold Sander-Nagashima, Die Bundesmarine 1950 bis 1972, Konzeption und Aufbau, München 2006, S. 15 - 426.

33 BA-MA, BW 2 / 9057, Fü S III 6, Vortragsnotiz für StAL Fü S III, Auftreten der STANAVFORLANT in der Ostsee, 19.2.73, mit weiteren Dokumenten.

34 Basisformationen zur RDJTF in: Jeffrey Record, The Rapid Deployment Force: Problems, Constraints, and Needs, in: The ANNALS of the American Academy of Political and Social Science, Vol. 457, No. 1, 109-120 (1981), Joint History Office (JCS), The History of the Unified Command Plan, 1946 - 1993 , Washington 1995, Part II., URL: http://www.dtic.mil/cgi-bin/GetTRDoc?AD $=$ ADA313508\&Location $=$ U2\&doc $=$ GetTRDoc.pdf., Paul K. Davis, Observation on the Rapid Deployment Joint Tast Force, Origins, Direction and Mission, Santa Monica 1982, URL: http://www.rand.org/pubs/papers/2005/P6751. pdf.

35 BA-MA, BW 2 / 13419, Verschlusssache. 
Für die NATO und insbesondere für Deutschland hatte das RDJTF-Konzept weitreichende Auswirkungen. ${ }^{36}$ Die Golfregion erhielt nun einen Status, der grundsätzlich dem europäischen Schauplatz gleichgeordnet, in mancherlei Hinsicht sogar übergeordnet wurde. Ausgehend von der Annahme, dass die Sowjetunion einen aggressiven Kurs im Nahen Osten verfolge und dort auch rasche Erfolge erzielen könne, glaubte die amerikanische Regierung massive Gegenmaßnahmen ergreifen zu müssen - nicht zuletzt wegen der strategischen Bedeutung der Ölfelder in der Region. Die Invasion in Afghanistan war dieser Lesart zufolge ein klares Indiz für die Absichten der Sowjetunion. Die massive Aufrüstung der sowjetischen Marine und deren zunehmende Präsenz auf den Weltmeeren, insbesondere auch dem Indischen Ozean, wurde als Auftakt für eine weitreichende Machtausdehnung gedeutet.

Da die Zahl der Verbündeten der USA in der Region abnahm und eine dauerhafte Präsenz von Truppen nicht in Frage kam, konzipierten die Vereinigten Saaten einen flexiblen Großverband auf Baukastenbasis, um schnell auf aggressive Akte reagieren zu können. Dabei gingen die Planer letztlich vom „worst-case“ aus (lokaler Krieg und gleichzeitiges Vorrücken der Sowjetunion von Norden). Die Eingreiftruppe konnte daher nicht lediglich aus einigen Bataillonen bestehen, wie etwa die AMF, sondern sollte sieben Divisionen mit den entsprechenden Luftwaffen- und Marinekomponenten umfassen.

Die entsprechenden Planungen gingen an die Substanz der NATO und erschütterten sie bis ins Mark. Die USA hatten, wohlweislich und aus jahrezehntelanger Erfahrung, nicht mit aktiver Beteiligung der Europäer gerechnet und ausschließlich US-Truppen eingeplant. Da jedoch eine Aufstockung der bestehenden US-Streitkräfte nicht in Frage kam, mussten die bereits bestehenden Einheiten für die RDJTF verwendet werden. Diese Einheiten waren für den Einsatz in Europa vorgesehen und hätten dort im Ernstfall nicht mehr zur Verfügung gestanden. SACEUR hätte bei einem Einsatz der RDJTF fast sämtliche strategischen Reserven verloren und wäre im Krisenfall der wichtigsten und kampfkräftigsten Einheiten, darunter auch Fallschirmjäger und amphibische Verbände, beraubt gewesen. ${ }^{37}$ Weit gravierender noch war die Tatsache, dass eine derartig große Truppe wie die RDJTF fast sämtliche strategischen Lufttransportkapazitäten benötigte. Überdies bedeutete die Verlagerung von Marineeinheiten und dem Transport zur See eine erhebliche Ausdünnung der eigenen Kräfte im Nordatlantik. Die Hauptschlagader der NATO, die Nachschublinien zwischen den USA und Europa, war dadurch in ihrer Existenz bedroht.

Unter der Reagan-Administration kam es insgesamt zu einer deutlichen Kräfteverschiebung. Der ganze Globus rückte nun in den Fokus, die Auseinandersetzung mit der Sowjetunion musste überall angenommen werden, ganz gleich, wie sich die Erfolgsaussichten jeweils darstellten. ${ }^{38}$ Da sich die ReaganRegierung bewusst war, dass weder die Europäer für ein offensives Engagement zu gewinnen waren noch die NATO-Grenzen erweitert werden konnten, verlangte sie kompensatorische Maßnahmen von den Europäern, also etwa die Ersetzung der

36 Zum Folgenden vgl. Stuart / Tow, Limits of Alliance, S. 88 - 99.

37 BA-MA, BW 2 / 14237, Verschlusssache.

38 BA-MA, BW 2 / 13419, Verschlusssache.
US-Truppen durch europäische Streitkräte, hier vor allem von Spezial- und Versorgungseinheiten, sowie durch politische und ökonomische Unterstützung bzw. Flankierung und die Gewährung von Überflugrechten.

Am bedeutsamsten aber war die Einplanung der US-Truppen außerhalb des NATO-Gebiets ohne Berücksichtigug der Einwände der Verbündeten. Die Europäer, insbesondere die Deutschen und hier vor allem auch die Spitzen der Streitkräfte, reagierten mit starker Ablehnung auf die amerikanischen Pläne. Man sah die gesamte Sicherheitsarchitektur gefährdet und die NATO in ihrer Existenz bedroht. ${ }^{39}$ Eine globale Interventionsstrategie mit Schwerpunkt auf dem Golf betrachteten einige Planer im Führungsstab der Streitkräfte als leichtsinniges "Cowboytum" auf dem Rücken der Europäer. Insbesondere befürchteten sie, dass ein Eingreifen der Amerikaner im Golf auf das NATO-Gebiet destabilisierend zurückwirken und hier zu Spannungen oder ernsten Krisen führen könnte. Sie verwiesen explizit darauf, dass die NATO ein regionales Bündnis sei. ${ }^{40}$ Washington betonte demgegenüber, dass der Schutz der Golfregion im ureigensten Interesse der NATO läge und entscheidende Gefahren für die Allianz gerade in der Vernachlässigung des Mittleren Ostens bestünden. Ferner wurde von amerikanischer Seite mit isolationistischen Tendenzen im US-Kongress argumentiert, die zu einem Rückzug der USA aus Europa führen könnten, wenn die europäischen NATO-Partner ihre eigenen Anstrengungen nicht erhöhten bzw. die USA nicht stärker unterstützten. ${ }^{41}$

Die amerikanische Regierung forderte als Lösung für die strategische Krise unter anderem die Verteidigungsausgaben stets auf dem Niveau der 3 Prozent-Klausel zu halten. ${ }^{42}$ Dies erinnerte an Argumente aus den 1960er Jahren, als die USA zur Absicherung der „Flexible Response“ verstärkte Anstrengungen der Europäer bei den konventionellen Streitkräften verlangt hatten.

Zwar fanden in dieser Phase eingehende Gespräche und Konsultationen sowie gemeinsame Planungen statt und wurden ausführliche Folgenabschätzungen erstellt und diskutiert, Einigung konnte jedoch nicht erzielt werden. Die Europäer, allen voran die Deutschen und die Briten, versuchten durch die Verankerung von Konsultationsrechten und strategische Beschränkungen (Vermeidung von „worst-case“-Szenarien) die Auswirkungen des RDJTF-Konzepts auf Europa so gering wie möglich zu halten, hatten damit aber kaum Erfolg. Die Vereinigten Staaten argumentierten, dass sie den Einsatz europäischer Truppen nicht verlangten und die Europäer den Amerikanern nicht vorschreiben könnten, wo ihre Streitkräfte zum Einsatz kämen. Dieses Argument sollte den Verbündeten zeigen, dass die USA zu militärischen Alleingängen bereit waren.

Mit dem RDJTF-Konzept waren die alten Kompromisse der 1960er Jahre, etwa der strategische Rotationsplan (etwa Übung „Big Lift“), hinfällig und in Europa wuchs die Furcht, es könne

39 BA-MA, BW 2 / 13419, Verschlusssache mit Begleitdokumenten.

40 BA-MA, BW 2 / 13419, Statements des Chairman des Military Committee und der Major NATO Commanders (MNC), Sachstand, 3.12.80.

41 BA-MA, BW 2 / 14237, Verschlusssache.

42 Gemeint war die Forderung, den Verteidigungshaushalt jährlich mindestens um drei - inflationsbereinigte - Prozent des Bruttoinlandproduktes zu erhöhen - eine Forderung, die die meisten NATO-Partner zumindest dauerhaft nicht erfüllen konnten. 
gegen andere Regionen ausgespielt werden. ${ }^{43}$ Am beunruhigsten war für die Europäer die Perspektive einer massiven Krise im Golf und die Verlagerung der RDJTF in diese Region bei gleichzeitigem Ausbruch einer Krise in Europa. Gerade die Bundesrepublik wäre dann durch eine Verlegung von US-Truppen und Lufttransportkapazitäten an den Golf in höchstem Maße gefährdet gewesen. Insofern bedrohte die RDJTF die Stabilität auch in Europa. ${ }^{44}$

Im Kern wirkten die alten Risse und Unvereinbarkeiten zwischen den USA und ihren europäischen Verbündeten fort, wurden durch die Situation im Nahen Osten lediglich neu generiert und verschärft. Das Regionalprinzip der NATO stand gegen das Globalprinzip der US-Regierung - Konfliktstrukturen, die heute noch gültig sind.

Verglichen mit der RDJTF war die AMF trotz der Ähnlichkeiten in der grundsätzlichen Auslegung (Mobilität, Lufttransport) in jedweder Hinsicht ein Zwerg. Zwischen den Einsatzgebieten beider Kontingente verlief eine unsichtbare Grenze, die zwei vollkommen unterschiedliche Welten trennte und in gewisser, nicht zu unterschätzender Weise bis heute trennt.

\section{Fazit}

Eine Einschätzung des Engagements der NATO außerhalb des Bündnisgebiets fällt ambivalent aus. Die NATO betrieb im Wesentlichen eine „non-policy“, wie ein Forscher dies einmal ausgedrückt hat. ${ }^{45}$ Es war dabei keineswegs von vornherein ausgeschlossen, dass die Allianz sich außerhalb Europas oder Nordamerikas engagierte oder sogar ihr Bündnisgebiet erweiterte. Auch hat sich die NATO immer wieder zu entsprechenden Problemen und Konflikten geäußert. Die Rückwirkungen von

43 BA-MA, BW 2 / 13419, Statements des Chairman des Military Committee und der Major NATO Commanders (MNC), Sachstand, 3.12.80.

44 BA-MA, BW 2 / 14237, Fü S III 6, Sprechzettel für Parlamentarischen Staatssekretär, USA-Reise, 2.9.82.

45 Frode Liland, Explaining NATO's Non-Policy on Out-Of-Area Issues during the Cold War, S. 188.
Krisen oder Kriegen auf die Allianz waren stets Gegenstand der Beratungen.

Es blieb indes weitgehend bei „Rückwirkungen“. Aktiv griffen zwar einzelne Mitglieder der NATO in Krisen oder Kriege jenseits des Bündnisgebiets ein, das Bündnis an sich beteiligte sich an solchen Aktionen jedoch nicht. Die These, dass diese Zurückhaltung die Existenz des Bündnisses über vierzig Jahre hinweg gerettet hat, ${ }^{46}$ ist mehr als einleuchtend. Die Konsequenzen hieraus für die Zeit nach dem Ende des Ost-West-Konflikts sind für die NATO jedoch nicht unbedingt positiv.

Seit 1990 hat sich vieles gewandelt. Auch die Bundesrepublik hat sich aktiv, inzwischen sogar mit Kampftruppen, auf europäischen und globalen Schauplätzen engagiert. Damit einher gingen teils einschneidende, vor allem organisatorische, strategische und rechtliche Veränderungen und Transformationen. Oberflächlich betrachtet, hat sich die NATO erheblich umgestaltet. ${ }^{47}$ Die Frage ist jedoch, ob sie sich auch im Kern, ihren inneren Strukturen und Prinzipien geändert hat. Nach den obigen Ausführungen fällt die Antwort hier negativ aus. Die tiefgreifenden Differenzen und Streitigkeiten innerhalb des Bündnisses haben sich trotz aller Konfliktbeilegungsmechanismen erhalten und sind seit den 1980er Jahren durch konkrete geografische, strategische, politische und ökonomische Probleme angereichert worden, die bis heute gültig sind: vor allem Regional- vs. Globalprinzip, die Gefahr amerikanischer Alleingänge, insbesondere im Mittleren Osten, Uneinigkeit über die einzusetzenden Mittel und die Lastenverteilung, Streit über die Frage der wechselseitigen Stabilisierung bzw. Destabilisierung zwischen Europa und dem Mittleren Osten. Das Problem der NATO ist nicht, dass sie ihr Gesicht seit 1990 gewandelt, sondern dass sie dies in zu geringem Ausmaß getan hat.

46 Stuart/Tow, Limits of Alliance, S. 313 - 322.

47 Vgl. dazu Johannes Varwick, Die NATO - Vom Verteidigungsbündnis zur Weltpolizei? München 2008, insbes. Kap. V - IX. Varwicks „phänomenologische“ Unterscheidung in drei verschiedene NATO-Allianzen (Kap. II) ist indes eher mechanistisch und verdeckt die bestehenden Kontinuitäten und Diskontinuitäten eher.

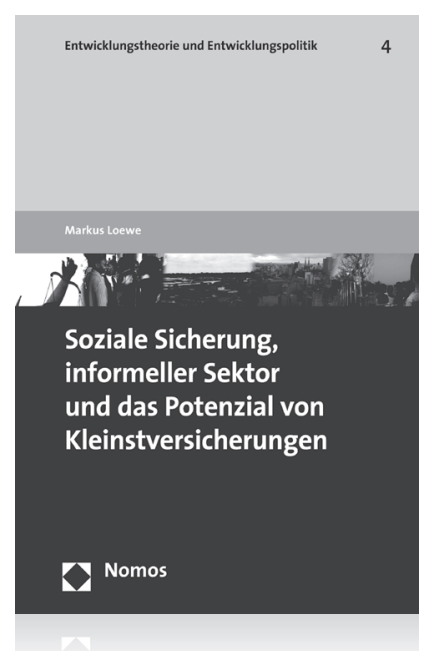

\section{Soziale Sicherung, informeller Sektor und das Potenzial von Kleinstversicherungen}

Von Markus Loewe

2009, 222 S., brosch., 44,- $€$ ISBN 978-3-8329-4017-1

(Entwicklungstheorie und Entwicklungspolitik, Bd. 4)

Wie gehen Menschen in Entwicklungsländern mit Risiken wie Krankheit, Alter oder Ernteausfall um? Warum haben Beschäftigte im informellen Sektor zumeist keinen Zugang zu effektiven Systemen der sozialen Sicherung? Welche Strategien sind geeignet, um ihre Lage zu verbessern? Welches Potenzial bergen Kleinstversicherungssysteme? Diese und zahlreiche andere Fragen diskutiert das vorliegende Buch. 